\title{
Cultural aspects of weaning practice in South Asian women in the UK
}

\author{
A. Skinner ${ }^{1}$, S. Smailes ${ }^{2}$, P. Raynor ${ }^{3}$, N. A. Small ${ }^{4}$, F. C. Dykes ${ }^{1}$ and N. M. Lowe ${ }^{1}$ \\ ${ }^{1}$ Food and Nutrition for Health Unit, University of Central Lancashire, Preston PR1 2HE, UK, ${ }^{2}$ Division of Psychology and \\ Social Change, Manchester Metropolitan University, Manchester M20 2RR, UK, ${ }^{3}$ Bradford Institute for Health Research, \\ Bradford Royal Infirmary, Bradford BD9 6RJ, UK and ${ }^{4}$ University of Bradford, Bradford BD5 OBB, UK
}

It has been suggested that South Asian (SA) women are abandoning nutritionally-superior indigenous weaning practices in favour of poorer British weaning practices ${ }^{(1)}$. The present study aims to explore the weaning practices of SA women living in Britain and the sources of information that affect their nutritional choices.

Two focus groups (FG) and seven individual interviews were conducted with twelve women of SA origin living in Bradford, UK in order to explore social and cultural beliefs surrounding food choices, including a discussion of the weaning process. The 2 FG consisted of four and five participants respectively and were held to develop ideas, highlight problems and facilitate the emergence of data that reflected participants' realities ${ }^{(2)}$. The FG and the interviews were transcribed verbatim and discussions classified into themes using quotes from the transcriptions ${ }^{(3)}$. In addition, information about sources of dietary information was collected by self-administered, tick box questionnaire from pregnant women as part of the Born in Bradford prospective cohort study at the Bradford Royal Infirmary (BRI). This cohort included women of all ethnicities attending the clinic for glucose tolerance testing at the BRI.

Preliminary analysis indicates that mothers found that the weaning recipes they received were bland and that the infants did not mature to develop a taste for their own cultural food. FG discussions revealed that, although mothers complied with some of the advice given to them such as feeding a low-salt diet, they also 'followed their own heart' to allow the infant to develop tastes belonging to their own culture.

A total of 1306 sets of questionnaire data were collected. Classified into ethnicity, 429 and 517 sets of valid data were collected from Asian or Asian British (SA) and Caucasian (C) groups respectively. Health visitors were the most popular choice as a source of nutritional knowledge among both the SA and C groups, with $38 \%$ and $28 \%$ respectively choosing this option. Family members, however, were more popular among the SA group than the $\mathrm{C}$ group, with $28 \%$ and $20 \%$ respectively choosing this option and $22 \%$ and $34 \%$ respectively choosing magazines, newspapers and books.

These data indicate that SA mothers value the advice given by health visitors; however, qualitative data suggest that there are problems integrating advice from health professionals with cultural norms. Weaning advice given should take into consideration traditional customs to inform good nutritional practices during the weaning process.

1. Condon L, Ingram J, Hamid N \& Hussein A (2003) Community Pract 76, 344-349.

2. Holloway I \& Wheeler S (1996) Focus groups. In Qualitative Research for Nurses, pp. 145-152. Edinburgh: Blackwell Science.

3. Polgar S \& Thomas SA (1991) Introduction to Research in the Health Sciences, 2nd ed., p. 127. Melbourne: Churchill Livingstone. 